\title{
Continuously tunable narrow-band terahertz generation with a dielectric lined waveguide driven by short electron bunches
}

\author{
T. H. Pacey® ${ }^{1,2,{ }^{*}}$ Y. Saveliev, ${ }^{1,2}$ A. Healy, ${ }^{3,2}$ P. G. Huggard $\odot,{ }^{4}$ B. Alderman, ${ }^{4}$ P. Karataev, ${ }^{5}$ \\ K. Fedorov $\oplus^{5,6}$ and G. Xia $\odot^{7,2}$ \\ ${ }^{1}$ ASTeC, STFC Daresbury Laboratory, Warrington, WA4 4AD, United Kingdom \\ ${ }^{2}$ Cockcroft Institute, Daresbury, Warrington, WA4 4AD, United Kingdom \\ ${ }^{3}$ Lancaster University, Lancaster, LA1 4YB, United Kingdom \\ ${ }^{4}$ RAL Space, STFC Rutherford Appleton Laboratory, Didcot OX11 OQX, United Kingdom \\ ${ }^{5}$ John Adams Institute at Royal Holloway, University of London, Egham, \\ Surrey TW20 OEX, United Kingdom \\ ${ }^{6}$ Tomsk Polytechnic University, 634050, Lenin Ave, Tomsk, Russia \\ ${ }^{7}$ The University of Manchester, Manchester, M13 9PL, United Kingdom
}

(Received 9 July 2019; published 12 September 2019)

\begin{abstract}
A continuously tunable narrow-band terahertz source based on Cherenkov wakefield generation in a dielectric lined waveguide driven by short sub-picosecond electron bunches has been experimentally demonstrated. A large tunability range of $0.55-0.95 \mathrm{THz}$ was achieved by implementing the waveguide design as a planar structure with variable $0.15-1.10 \mathrm{~mm}$ gap and thin $25 \mu \mathrm{m}$ dielectric layers. The source operates essentially in a single mode regime with higher order modes of negligible intensity. THz pulse energy was measured across the tunable range and compared with theory.
\end{abstract}

DOI: 10.1103/PhysRevAccelBeams.22.091302

Dielectric lined waveguides (DLW) driven by short electron bunches have been demonstrated to be powerful sources of $\mathrm{THz}$ radiation. The underlying physical mechanism is coherent Cherenkov radiation (CCR) that takes place when the bunch length is shorter than the radiation wavelength [1]. Such short sub-picosecond electron bunches are now routinely generated by modern photoinjector based accelerators. Cylindrical DLWs in the form of a dielectric capillary with outer metallic coating are normally considered for CCR generation [2-5]. Coupling of radiation into free space is achieved either by horn [2] or via an angled cut in the capillary waveguide [6,7]. Metallic corrugated structures sharing similar physical principles with DLWs are also used for generation of $\mathrm{THz}$ radiation [8-10].

Unlike other methods of generating high power $\mathrm{THz}$ radiation (laser based $[11,12]$, coherent transition radiation (CTR) based [13] etc.), the CCR sources are essentially narrow-band devices operating in single- or multimode regimes. This advantage ideally should be complimented by the provision of frequency tunability. Stepwise tunability can be achieved by interchanging cylindrical DLWs

\footnotetext{
*thomas.pacey@stfc.ac.uk

Published by the American Physical Society under the terms of the Creative Commons Attribution 4.0 International license. Further distribution of this work must maintain attribution to the author(s) and the published article's title, journal citation, and DOI.
}

with different frequency spectra [2]. Resonant excitation of different CCR modes by bunch trains in multimode structures has also been studied [3]. Continuous tunability with DLW with a ferroelectric layer has been demonstrated, albeit in a very narrow range [14]. With a low energy (few $\mathrm{MeV}$ ) electron beam, some continuous tunability can be attained through varying the beam energy as demonstrated in [10] using a fixed gap metal corrugated waveguide.

Planar DLW and metallic corrugated waveguides are normally considered as accelerating structures allowing for a greater transverse stability of the electron beams $[15,16]$, or as energy dechirpers that increase efficiency of free electron lasers $[17,18]$. The rectangular DLW can also be employed as continuously tunable narrow-band $\mathrm{THz}$ source with the provision of variable structure gap and correct choice of dielectric liner. As follows from the wakefield theory of planar dielectric structures [19], DLWs with thick dielectric layers, more than 100s of $\mu \mathrm{m}$, exhibit CCR spectra with multiple modes and very low frequency dependence on structure gap. In contrast, DLWs with thin dielectric layers of a few 10s of $\mu$ m operate effectively in a single mode regime with high tunability as a function of the structure gap. The latter is the subject of the experimental investigation presented in this paper.

The rectangular DLW designed for these experiments was $2 \mathrm{~mm}$ wide and $60 \mathrm{~mm}$ long overall and is shown schematically in Fig. 1. The dielectric layer was $40 \mathrm{~mm}$ long and made of $25 \mu \mathrm{m}$ thick quartz plates with assumed dielectric permittivity of $\epsilon_{r}=3.8$. The dielectric plates 
were recessed into copper substrates leaving the first $10 \mathrm{~mm}$ of the structure to act as a beam collimator. Several options for the output coupling antenna were simulated in CST Microwave Studio and a simple horn of $10 \mathrm{~mm}$ long and $12^{\circ}$ half angle opening in the vertical $(y)$ plane was found to be satisfactory in terms of gain and directivity. The radiation was emitted in two lobes with the same forward angle as the angle of the taper. The structure tunability caused only a weak decrease in gain with increasing structure gap. The gap (2a) between the quartz plates was controlled by two in vacuum picomotors and was evaluated using high resolution CCD cameras. This method resulted in a precision of $\pm 20 \mu \mathrm{m}$ in setting the structure gap.

Experiments were conducted on CLARA/VELA facility at Daresbury Laboratory, STFC [20]. An electron beam with $10 \mathrm{~Hz}$ bunch repetition frequency was generated in the $3 \mathrm{GHz}$ RF photoelectron gun of the CLARA front end followed by a normal conducting linac. The $35 \mathrm{MeV}$ electron beam was then injected into the VELA beamline via a short dog-leg section where longitudinal compression of the electron bunch from $\approx 2$ ps down to $\approx 0.3$ ps RMS took place. Bunch compression could be varied by changing the linac off-crest accelerating phase. The beam was transported to an experimental area equipped with large $2.3 \mathrm{~m}$ long vacuum chamber and a set of standard diagnostics (energy spectrometer, YAG screens, and beam position monitors). Transverse beam size at the interaction point was typically $70-100 \mu \mathrm{m}$ RMS at bunch charges of up to $100 \mathrm{pC}$. The DLW structure was mounted on a multiaxis in-vacuum motorized assembly to ensure its transverse position and angular alignment to the electron beam.

The $\mathrm{THz}$ radiation measurement system is shown schematically in Fig. 1. CCR was collected using an off-axis parabolic (OAP) mirror with a hole at the center for the electron beam to pass through. The OAP mirror was positioned such that the output of the DLW was at its focal point $(101.6 \mathrm{~mm})$. The OAP collimated the radiation and transported it out of the vacuum chamber through a z-cut quartz window. The radiation was either focussed by a TPX lens onto the pyroelectric detector for direct measurement of the pulse energy, or transported further to a Martin-Puplett interferometer (MPI) [21]. The MPI is operationally similar to a Michelson interferometer used in a number of other CCR studies, but uses wire grid polarizers as beam splitters for increased efficiency and therefore requires the input radiation to be polarized. As the CCR from our DLW is polarized vertically, along the variable gap axis (y), the MPI accepts the radiation through the input polarizer without substantial losses. It is worth noting that previous similar experiments [2,3] have employed liquid helium cooled bolometers for $\mathrm{THz}$ detection. It was established in these experiments that pyroelectric detectors (Gentec THZ5I-BL-BNC-D0) had sufficiently high sensitivity and signal to noise ratio for interferometric measurements.

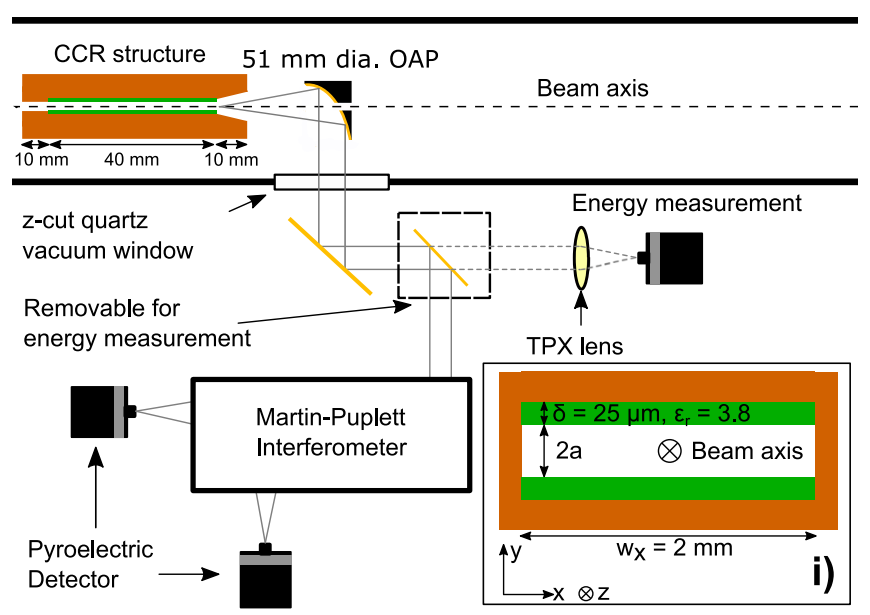

FIG. 1. Experimental setup. The CCR was collected by a $51 \mathrm{~mm}$ diameter off axis parabolic (OAP) mirror with a hole to pass the electron beam. TPX lenses were used for focusing of the CCR onto the detectors. All mirrors were gold coated and polished to optical finish. An additional pyroelectric detector (not shown) was used for shot-to-shot subtraction of environmental noise. Inset i), cross-section of the DLW with coordinate system and parameter notation.

Two techniques were employed to increase the signal to noise ratio from the pyroelectric detectors. First, a third pyroelectric detector was used to record and subtract the environmental background noise on a shot-by-shot basis. Second, multishot averaging (50 successive shots) was used to accurately resolve the signal at low pulse energies and in the interferograms, resulting in a detector noise floor of $\leq 2 \mathrm{mV}$. Detectors were placed in both output arms of the MPI and the interferogram was normalised to the signal difference divided by the signal sum [22].

The CCR is excited as a set of discrete longitudinal section magnetic (LSM) and longitudinal section electric (LSE) wakefield modes in the DLW by the electron beam. The dispersion relation for the $\mathrm{LSM}_{m, n}$ modes [19] is:

$$
\operatorname{coth}\left(k_{x, m} a\right) \cot \left(k_{y, n} \delta\right)-\frac{k_{y, n}}{\epsilon_{r} k_{x, m}}=0,
$$

with $k_{x, m} \equiv(2 m+1) \frac{\pi}{w_{x}}$ (we consider only odd modes, $m=0,1,2$, because even modes do not couple efficiently to the electron beam in a symmetric system) and $x, y$ defined in Fig. 1. The mode frequencies, $f_{m, n}$ are given by

$$
f_{m, n}^{2}=\frac{c^{2}}{4 \pi^{2}}\left(\frac{k_{x, m}^{2}+k_{y, n}^{2}}{\epsilon_{r}-1}\right) .
$$

Following the notation of [19], the lowest order wakefield mode is $\mathrm{LSM}_{0,0}$ (also referred to as $\mathrm{LSM}_{1,1}$ in [23] and other published studies), with $k_{y, 0}$ given by the first solution to Eq. (1). For brevity we denote the fundamental CCR mode $\left(\mathrm{LSM}_{0,0}\right)$ frequency as $f_{0}$. The contribution of longitudinal 

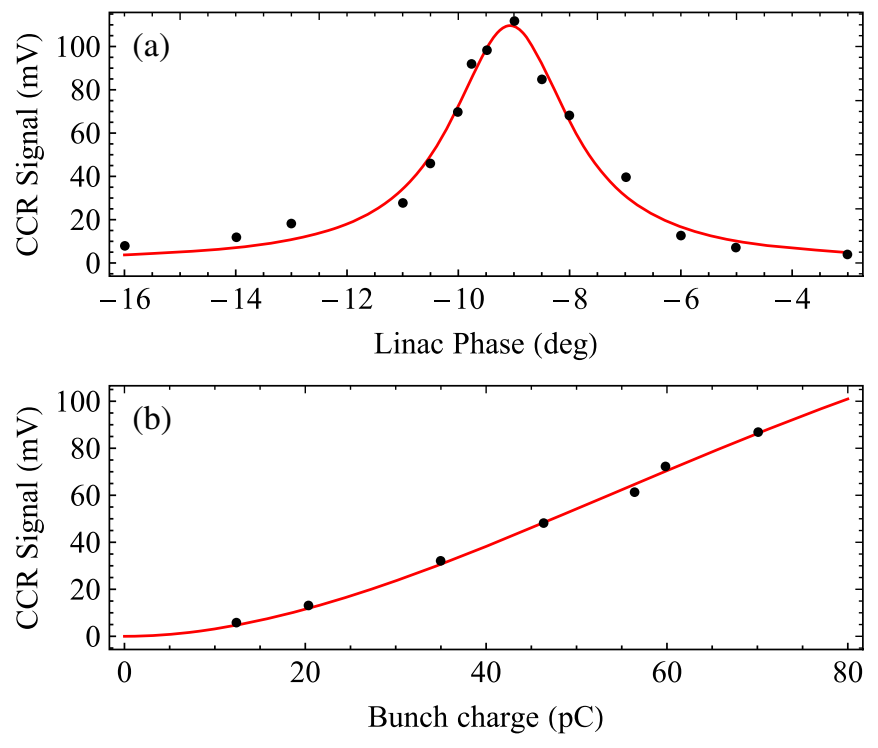

FIG. 2. Variation of CCR pulse energy with machine parameters. (a) CCR signal as a function of the linac off-crest phase at bunch charge of $90 \mathrm{pC}$ and $2 a=540 \mu \mathrm{m}$. (b) Dependence of the CCR energy on bunch charge at maximal bunch longitudinal compression; $2 a=590 \mu \mathrm{m}$.

section electric (LSE) modes is negligible due to the high structure aspect ratio, i.e., $w_{x}>2 a$.

The total emitted CCR energy depends strongly on the bunch length that, in turn, is determined by the linac offcrest phase as demonstrated in Fig. 2(a). A coherent transition radiation (CTR) target was installed alongside the DLW structure to evaluate the electron bunch length using the MPI and to confirm the machine setup reproducibility on a daily basis by measuring the CTR total power as a function of the linac phase. The RMS bunch length, $\sigma_{t}$, of 0.3 ps at maximum compression with the linac set to $-9^{\circ}$ off-crest phase was measured using an established CTR interferogram fitting technique [24,25]. Longer bunch lengths were evaluated from induced energy modulation along the bunch similar to that reported in [26]. At $-16^{\circ}$ off-crest phase, the bunch length at $10 \%$ level was estimated as 3.3 ps using the modulation method. The coherent nature of CCR generation mechanism was confirmed by the enhancement of the signal as the bunch was compressed, see Fig. 2(a).

The variation of CCR signal, $I$, with bunch charge, $Q$, is shown in Fig. 2(b). To minimize the effect of space charge on the longitudinal beam dynamics, the bunch charge was varied by occluding the beam in the injector of the accelerator before compression in the dog-leg section; instead of varying the photoinjector laser power. However, computer simulations indicate that the length of the bunch arriving to experimental area still depends slightly on the bunch charge and this may account for the observation that the dependence of CCR energy on bunch charge does not follow a pure quadratic function. The experimental data in Fig. 2(b) was fit with the
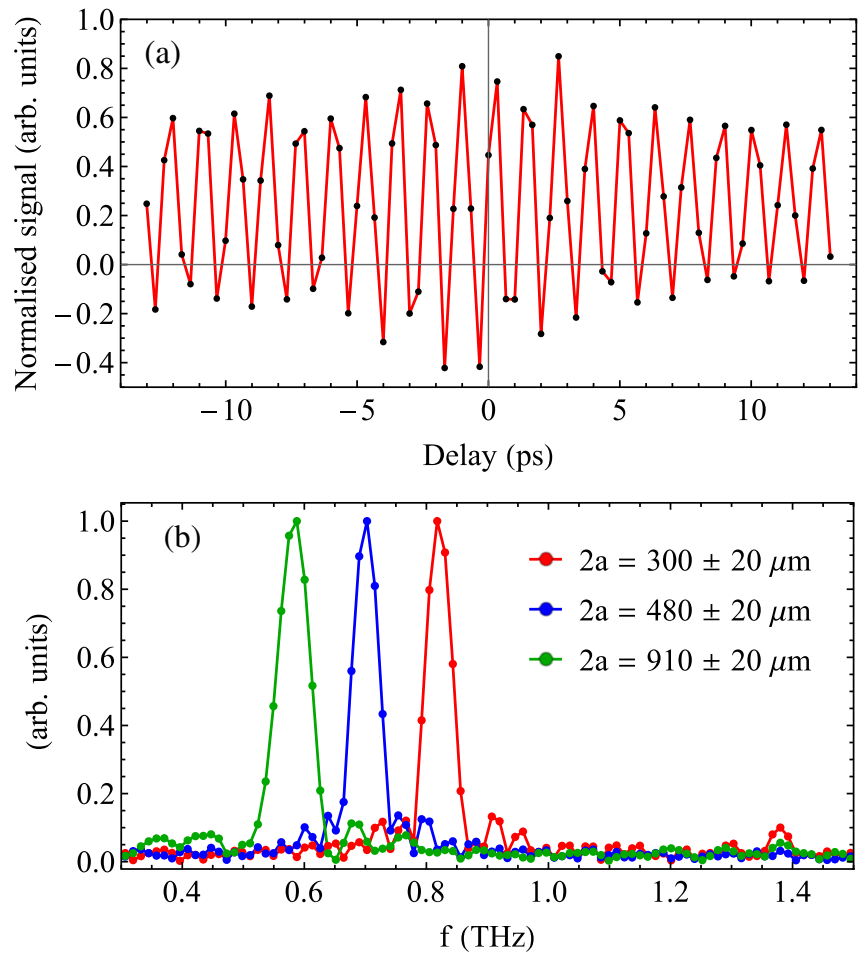

FIG. 3. (a) Interferogram for structure gap $2 a=300 \mu \mathrm{m}$. (b) Example CCR power spectra calculated from corresponding interferograms for structure gaps of $300 \mu \mathrm{m}, 480 \mu \mathrm{m}$, and $910 \mu \mathrm{m}$.

expression $I \propto Q^{2}\left|F\left(\sigma_{t}\right)\right|^{2}$, with the Gaussian form factor $F \propto \exp \left(-2 \pi^{2} \sigma_{t}^{2} f_{0}^{2}\right)$. The frequency $f_{0}$ was calculated from Eq. (2) and $\sigma_{t}$ was varied linearly with charge from $0.30 \mathrm{ps}$ at $70 \mathrm{pC}$ to $0.25 \mathrm{ps}$ at $10 \mathrm{pC}$. The assumption of a Gaussian bunch profile is corroborated by the fact that the variation of RMS bunch length in fitting is in agreement to that measured experimentally using CTR. Beam dynamics simulations also predict the bunch profile should be quasi-Gaussian at maximum compression.

The pyroelectric detector used in the pulse energy measurements presented in Fig. 2(b) was not directly calibrated in the $\mathrm{THz}$ frequency range. The detector responsivity was estimated via calibration with a $532 \mathrm{~nm} \mathrm{CW}$ diode laser chopped at $10 \mathrm{~Hz}$ with $1.5 \%$ duty cycle and assuming the absorption spectrum as that given by the manufacturer data for this detector. The maximum observed signal of $110 \mathrm{mV}$ in Fig. 2(a) was found to correspond to a THz pulse energy of approximately $0.3 \mu \mathrm{J}$. The losses from the quartz window were independently measured to be $\approx 50 \%$. Disregarding other potential transport losses that were largely unknown, the pulse energy at source exit can be estimated as exceeding $0.6 \mu \mathrm{J}$.

A representative interferogram registered at $300 \mu \mathrm{m}$ gap and corresponding CCR spectrum are shown in Fig. 3. Figure 3(b) also shows spectra at 480 and $910 \mu \mathrm{m}$ gaps. The interferograms were collected by averaging the response of the pyroelectric detector for 50 successive shots at each delay step. The standard error for each data point is smaller than 

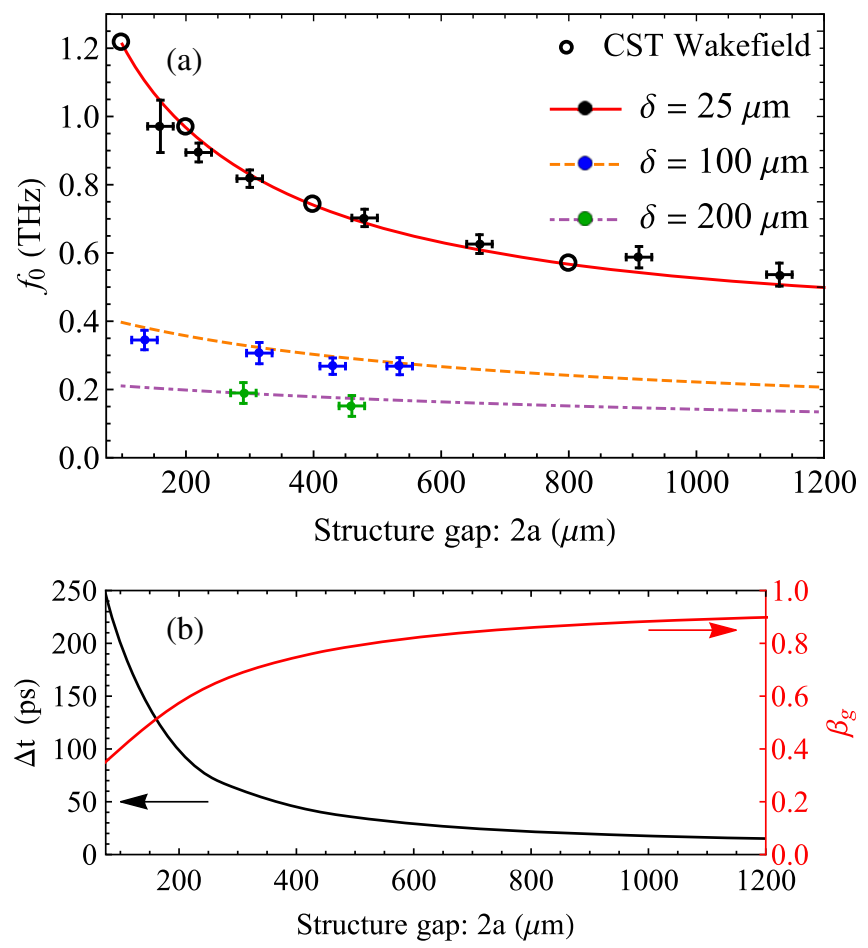

FIG. 4. (a) Measured frequency of the fundamental mode as a function of the structure gap at three dielectric thicknesses of 25, 100, and $200 \mu \mathrm{m}$. Vertical error bars represent FWHM width of spectral lines. Solid lines are solutions of the dispersion relation in Eq. (1). (b) Calculated CCR group velocity, relative to the speed of light in vacuum, and corresponding CCR pulse length [Eq. (3)] as a function of the structure gap for the case of $25 \mu \mathrm{m}$ thick dielectric.

the size of the point marker. The CCR spectra were calculated from fast Fourier transform of the interferograms without the use of apodization or zero-padding to smooth and increase the apparent spectral resolution. Broadband coherent diffraction radiation (CDR) could make a significant contribution to the CCR spectra [9]. This was not investigated in detail but the absence of a peak at 0 ps delay in Fig. 3(a) clearly indicates that the contribution of CDR is negligible due to our experimental configuration, similar to [2], with output horn at the exit of the DLW and the fact that CDR from the OAP hole is largely lost during transport to the MPI.

The continuous tunability of the CCR source under investigation with thin $\delta=25 \mu \mathrm{m}$ dielectric is demonstrated in Fig. 4(a). The fundamental mode frequency varies from 0.55 to $0.95 \mathrm{THz}$, as the gap of the DLW structure varies from 150 to $1100 \mu \mathrm{m}$. For comparison, experimental data obtained with quartz layers of $100 \mu \mathrm{m}$ and $200 \mu \mathrm{m}$ thickness and the same planar geometry of the DLW structure is also given in Fig. 4(a). As expected, the tunability of structures with thicker dielectrics was greatly reduced and the intensity of HOMs was higher. The measured frequencies agree, within experimental errors, with the theoretical predictions from Eq. (2). The analytically calculated frequencies are corroborated by CST Wakefield
Solver simulations that are shown with open circles in Fig. 4(a). Higher order modes (HOMs) are noticeable in the CCR spectra, with horizontal HOMs $\left(\mathrm{LSM}_{1,0}\right)$ excited at frequencies approximately $0.1 \mathrm{THz}$ higher than the fundamental mode. Vertical HOMs were also excited, in the frequency range 1.2 to $1.4 \mathrm{THz}\left(\mathrm{LSM}_{0,1}\right)$. All HOMs exhibited very low intensity that could be further reduced in amplitude by increasing the transverse electron beam aspect ratio from $1: 1$ (horizontal to vertical) to $5: 1$, as was observed in these experiments.

Vertical error bars shown in Fig. 4(a) represent FWHM values of the measured spectral lines [Fig. 3(b)] that amount to 50-60 GHz across the whole DLW gap range with the exception of the smallest gap of $140 \mu \mathrm{m}$ at which the FWHM is approximately $150 \mathrm{GHz}$. At this gap, the signal to noise ratio was low in the interferogram thus affecting accuracy of the spectrum calculation. With the limited interferometer stage travel range used in Fig. 3(a), the lower limit of the measured width of the spectral lines is $\approx 50 \mathrm{GHz}$. In reality, the bandwidth of the spectral line should vary significantly over the tunability range of the DLW. This can be estimated from the time-bandwidth product assuming a rectangular envelope of the $\mathrm{THz}$ pulse. The radiation pulse length is given by

$$
\Delta t=L \frac{1-\beta_{g}}{\beta_{g} c}
$$

where $L$ is the structure length and $\beta_{g}$ is the group velocity of the $\mathrm{LSM}_{0,0}$ mode (normalized to $c$ ) which, in turn, is calculated from the dispersion relation in [23]. Both the group velocity and the pulse length are presented in Fig. 4(b) as a function of the structure gap. At larger gaps of $\approx 1000 \mu \mathrm{m}$, the minimum theoretical bandwidth is expected to be 50-70 GHz which is consistent with the measurements [Fig. 4(b)]. At lower gaps, $2 a<200 \mu \mathrm{m}$, the minimal bandwidth should reduce to below $10 \mathrm{GHz}$. Dielectric and waveguide losses and dispersion within the structure would also affect the actual bandwidth of the radiation. Investigation of these effects and improvement of the interferometer spectral resolution is the subject of further studies.

The CCR pulse energy varied significantly across the DLW tunability range as shown in Fig. 5. The absorption spectrum of the pyroelectric detector is approximately flat across the frequency range of Fig. 5, as follows from the data provided by the manufacturer. These measurements were made with the pyroelectric detector within the interferometer as opposed to direct measurements in Fig. 2. The electron beam in these measurements had a finite vertical size of $\sigma_{y}=70 \mu \mathrm{m}$ that caused effective beam losses through the structure with gaps lower than $500 \mu \mathrm{m}\left(f_{0}>0.7 \mathrm{THz}\right)$. Assuming a Gaussian transverse distribution and quadratic dependence of the CCR pulse energy on bunch charge, the experimental data in Fig. 5 (red dots) was adjusted to show the expected signals without beam losses (black dots). The detector peak signals are plotted against frequency of 


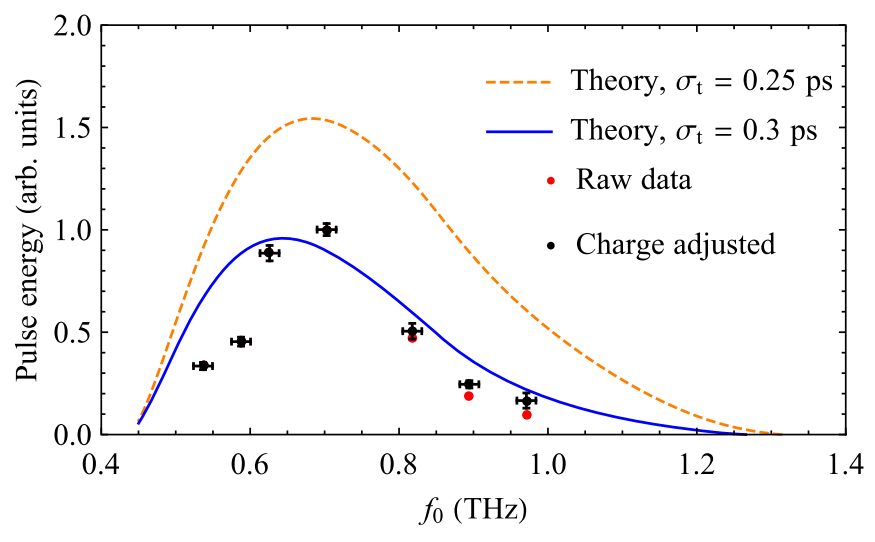

FIG. 5. Variation of CCR pulse energy as a function of fundamental frequency. The data (red dots) is also adjusted for loss of charge due to finite transverse electron beam size (black dots). Solid and dotted lines are theoretical dependencies for two bunch lengths of $0.30 \mathrm{ps}$ and $0.25 \mathrm{ps}$.

radiation using the measured relation between the structure gap and frequency as shown in Fig. 4(a). Maximal CCR power was registered at $f_{0} \approx 0.65 \mathrm{THz}$. Reduction of power at lower frequencies can be attributed primarily to the lower strength of wakefields at larger structure gaps $[19,23]$. At higher frequencies, the dominant factor would be a reduced coupling into the fundamental mode due to the finite length of the electron bunch. To estimate theoretically the CCR pulse energy as a function of structure gap (and therefore frequency), the both relative power and pulse length of the LSM mode were calculated. The relative power of the $\mathrm{LSM}_{0,0}$ mode as a function of structure gap was calculated from the 2D Poynting vector integrated across the waveguide cross section. This accounts for both relative spatial variation and amplitude variation of the fields as a function of structure gap. The equations used are given in [19]. The pulse length was calculated from Eq. (3), using the variation of $\beta_{g}$ with structure gap [Fig. 4(b)]. To account for coupling of the bunch to the mode with frequency $f_{0}$, a Gaussian form factor was used with $\sigma_{t}=0.3 \mathrm{ps}$. This expression was normalized to fit the measured power as a function of structure gap. Equations (1) and (2) were used to write the final expression as a function of CCR frequency $f_{0}$. The measured data in Fig. 5 is plotted against the measured frequency [Fig. 4(a)], hence the discrepancy at low frequencies $(0.5$ to $0.6 \mathrm{THz})$ where the measured CCR frequency was higher than the theoretical predication. To demonstrate the sensitivity of the CCR power to bunch length the prediction with $\sigma_{t}=0.25 \mathrm{ps}$ is included in Fig. 5.

Whilst this source requires a relatively high energy electron beam to produce $\mathrm{THz}$ radiation, it should be considered as part of the overall accelerator facility adding a highly compact and cost effective component. In the context of an accelerator facility, this source benefits strongly from intrinsic synchronisation to the electron beam, which is an advantage for pump-probe type experiments.
This tunable source could be incorporated into relatively compact and cheap accelerator based $\mathrm{THz}$ sources similar to [10]. Alternatively, it could be incorporated into future high energy laser plasma accelerators, which are capable of producing high energy ultrashort electron beams suitable for CCR generation.

A number of $\mathrm{THz}$ applications would require high instantaneous radiation power as well as high pulse energy. These requirements may not be fully satisfied at high frequency end, $>0.8 \mathrm{THz}$, of the tunability range because of reduced pulse energy [Fig. 5] and increased pulse length [Fig. 4(b)]. The former can be alleviated by decreasing bunch length to sub-100 fs levels and increasing the bunch charge to nano-Coulomb range. Both parameters can be achieved with modern accelerators. Significant increase in THz energy per pulse with shorter bunches is illustrated in Fig. 5 where theoretical predictions of the pulse energy are given for bunch lengths of $0.3 \mathrm{ps}$ and only slightly reduced $0.25 \mathrm{ps}$. The CCR pulse length can be reduced, and instantaneous power increased, by introducing a frequency chirp along the pulse followed by a dispersive pulse compressor as discussed theoretically in [27]. With planar DLW structures, this chirp could be readily implemented by making the structure gap longitudinally tapered, with the taper also being tunable.

With the DLW parameters used in these experiments, the tunability range is limited at both high and low frequency ends. At high frequencies, the limitation stems from decreased coupling between the beam and wakefield and increased beam losses due to the smaller structure gaps required. The low frequency limit is effectively governed by the dielectric thickness and occurs when no further frequency change takes place while varying the structure gap [Fig. 4(a)]. For the structure used this occurs at $2 a>2 \mathrm{~mm}$ with a fundamental frequency of $f_{0}=0.45 \mathrm{THz}$. Extending the frequency range beyond demonstrated in these experiments is feasible by manipulating the DLW parameters, primarily dielectric thickness $\delta$ and the dielectric constant of the material. For example, decreasing $\delta$ to $\sim 10 \mu \mathrm{m}$ and below can extend the frequency to $2-3 \mathrm{THz}$. This however can be achieved only at the expense of the reduced $\mathrm{THz}$ pulse energy, which can be offset by reduction of the bunch length and transverse beam size. Detailed analysis of these options is the subject of further investigation.

In conclusion, we have demonstrated continuously tunable $\mathrm{THz}$ coherent Cherenkov radiation from a planar dielectric lined waveguide with variable gap driven by sub-ps electron beam in a wide frequency range of $0.55-0.95 \mathrm{THz}$. The strong agreement demonstrated between experimental results and theoretical predictions - across a wide range of dielectric thicknesses and gaps validates these analytical models, which can be used in the design of both CCR sources and future DWA accelerators. With the thin $25 \mu \mathrm{m}$ dielectric layers employed, the output radiation is narrow-band and predominantly in a single fundamental mode. Excited higher order modes are of negligible intensity. The $\mathrm{THz}$ 
pulse energy varies across the frequency range due to variation in the structure gap and coupling coefficient to a fundamental structure mode peaking at $f_{0} \approx 0.7 \mathrm{THz}$. Given the relatively small bunch charge of 70-90 pC available for these experiments and largely unknown losses of the quasioptical $\mathrm{THz}$ transport, we did not aim to demonstrate high levels of $\mathrm{THz}$ pulse energy and estimate it to be a fraction of a micro-Joule across the tunability range.

The authors thank CLARA/VELA commissioning team for preparation of the accelerator for these experiments and Mark Surman at STFC for help with THz diagnostics.

[1] J. E. Walsh, T. C. Marshall, and S. P. Schlesinger, Generation of coherent cerenkov radiation with an intense relativistic electron beam, Phys. Fluids 20, 709 (1977).

[2] A. M. Cook, R. Tikhoplav, S. Y. Tochitsky, G. Travish, O. B. Williams, and J. B. Rosenzweig, Observation of NarrowBand Terahertz Coherent Cherenkov Radiation from a Cylindrical Dielectric-lined Waveguide, Phys. Rev. Lett. 103, 095003 (2009).

[3] G. Andonian, O. Williams, X. Wei, P. Niknejadi, E. Hemsing, J. B. Rosenzweig, P. Muggli, M. Babzien, M. Fedurin, K. Kusche, R. Malone, and V. Yakimenko, Resonant excitation of coherent cerenkov radiation in dielectric lined waveguides, Appl. Phys. Lett. 98, 202901 (2011).

[4] K. Kan, J. Yang, A. Ogata, T. Kondoh, K. Norizawa, and Y. Yoshida, Multimode terahertz-wave generation using coherent cherenkov radiation, Appl. Phys. Lett. 99, 231503 (2011).

[5] S. Antipov, S. Baryshev, C. Jing, P. Schoessow, A. Kanareykin, M. Fedurin, C. Swinson, D. Wang, A. Zholents, and W. Gai, High power terahertz radiation source based on electron beam wakefields, AIP Conf. Proc. 1777, 070003 (2016).

[6] S. N. Galyamin, A. V. Tyukhtin, S. Antipov, and S. S. Baturin, Terahertz radiation from an ultra-relativistic charge exiting the open end of a waveguide with a dielectric layer, Opt. Express 22, 8902 (2014).

[7] S. Antipov, S. V. Baryshev, R. Kostin, S. Baturin, J. Qiu, C. Jing, C. Swinson, M. Fedurin, and D. Wang, Efficient extraction of high power thz radiation generated by an ultra-relativistic electron beam in a dielectric loaded waveguide, Appl. Phys. Lett. 109, 142901 (2016).

[8] A. Smirnov, R. Agustsson, S. Boucher, T. Grandsaert, J. Hartzell, M. Ruelas, S. Storms, A. Andrews, B. Berls, C. Eckman, K. Folkman, A. Hunt, Y. Kim, A. KnowlesSwingle, C. O’Neill, M. Smith, and P. Buaphad, Development of a Compact Insertion Device for Coherent Sub-mm Generation, in Proceedings, 4th International Particle Accelerator Conference (IPAC 2013): Shanghai, China, May 12-17 (JACoW, Shanghai, China, 2013), pp. 22952297.

[9] K. Bane, G. Stupakov, S. Antipov, M. Fedurin, K. Kusche, C. Swinson, and D. Xiang, Measurements of terahertz radiation generated using a metallic, corrugated pipe, Nucl. Instrumen. Methods Phys. Res., Sect. A 844, 121 (2017).
[10] A. V. Smirnov, R. Agustsson, W. J. Berg, S. Boucher, J. Dooling, T. Campese, Y. Chen, L. Erwin, B. Jacobson, J. Hartzell, R. Lindberg, A. Murokh, F. H. O'Shea, E. Spranza, S. Pasky, M. Ruelas, N. S. Sereno, Y. Sun, and A. A. Zholents, Observation of a variable sub-thz radiation driven by a low energy electron beam from a thermionic rf electron gun, Phys. Rev. Accel. Beams 18, 090703 (2015).

[11] G. Liao, Y. Li, H. Liu, G. G. Scott, D. Neely, Y. Zhang, B. Zhu, Z. Zhang, C. Armstrong, E. Zemaityte, P. Bradford, P. G. Huggard, D. R. Rusby, P. McKenna, C. M. Brenner, N. C. Woolsey, W. Wang, Z. Sheng, and J. Zhang, Multimillijoule coherent terahertz bursts from picosecond laserirradiated metal foils, Proc. Natl. Acad. Sci. U.S.A. 116, 3994 (2019).

[12] M. J. Cliffe, D. M. Graham, and S. P. Jamison, Longitudinally polarized single-cycle terahertz pulses generated with high electric field strengths, Appl. Phys. Lett. 108, 221102 (2016).

[13] X. Yang, E. Brunetti, and D. A. Jaroszynski, High-energy coherent terahertz radiation emitted by wide-angle electron beams from a laser-wakefield accelerator, New J. Phys. 20, 043046 (2018).

[14] C. Jing, A. Kanareykin, J. G. Power, M. Conde, W. Liu, S. Antipov, P. Schoessow, and W. Gai, Experimental Demonstration of Wakefield Acceleration in a Tunable Dielectric Loaded Accelerating Structure, Phys. Rev. Lett. 106, 164802 (2011).

[15] T. C. Marshall, C. Wang, and J. L. Hirshfield, Femtosecond planar electron beam source for micron-scale dielectric wake field accelerator, Phys. Rev. Accel. Beams 4, 121301 (2001).

[16] G. Andonian, D. Stratakis, M. Babzien, S. Barber, M. Fedurin, E. Hemsing, K. Kusche, P. Muggli, B. O'Shea, X. Wei, O. Williams, V. Yakimenko, and J. B. Rosenzweig, Dielectric Wakefield Acceleration of a Relativistic Electron Beam in a Slab-Symmetric Dielectric Lined Waveguide, Phys. Rev. Lett. 108, 244801 (2012).

[17] S. Antipov, S. Baturin, C. Jing, M. Fedurin, A. Kanareykin, C. Swinson, P. Schoessow, W. Gai, and A. Zholents, Experimental Demonstration of Energy-Chirp Compensation by a Tunable Dielectric-based Structure, Phys. Rev. Lett. 112, 114801 (2014).

[18] Z. Zhang, K. Bane, Y. Ding, Z. Huang, R. Iverson, T. Maxwell, G. Stupakov, and L. Wang, Electron beam energy chirp control with a rectangular corrugated structure at the linac coherent light source, Phys. Rev. Accel. Beams 18, 010702 (2015).

[19] D. Mihalcea, P. Piot, and P. Stoltz, Three-dimensional analysis of wakefields generated by flat electron beams in planar dielectric-loaded structures, Phys. Rev. ST Accel. Beams 15, 081304 (2012).

[20] P. McIntosh, D. Scott, S. Jamison, B. Fell, P. Williams, T. Jones, Y. Saveliev, N. Thompson, L. Cowie, B. Militsyn et al., The vela and clara test facilities at daresbury laboratory, in Proc. 28th Linear Accelerator Conf. (LINAC'16) (JACow, East Lansing, MI, 2016), pp. 734739, https://doi.org/10.18429/JACoW-LINAC2016-TH3 A03. 
[21] D. Martin and E. Puplett, Polarised interferometric spectrometry for the millimetre and submillimetre spectrum, Infrared Phys. 10, 105 (1970).

[22] L. Frohlich, Bunch length measurements using a MartinPuplett interferometer at the VUV-FEL, Ph.D. thesis, Hamburg U., 2005.

[23] L. Xiao, W. Gai, and X. Sun, Field analysis of a dielectricloaded rectangular waveguide accelerating structure, Phys. Rev. E 65, 016505 (2001).

[24] A. Murokh, J. Rosenzweig, M. Hogan, H. Suk, G. Travish, and U. Happek, Bunch length measurement of picosecond electron beams from a photoinjector using coherent transition radiation, Nucl. Instrumen. Methods Phys. Res., Sect. A 410, 452 (1998).
[25] I. Nozawa, K. Kan, J. Yang, A. Ogata, T. Kondoh, M. Gohdo, K. Norizawa, H. Kobayashi, H. Shibata, S. Gonda, and Y. Yoshida, Measurement of $<20 \mathrm{fs}$ bunch length using coherent transition radiation, Phys. Rev. Accel. Beams 17, 072803 (2014).

[26] S. Antipov, C. Jing, M. Fedurin, W. Gai, A. Kanareykin, K. Kusche, P. Schoessow, V. Yakimenko, and A. Zholents, Experimental Observation of Energy Modulation in Electron Beams Passing through Terahertz Dielectric Wakefield Structures, Phys. Rev. Lett. 108, 144801 (2012).

[27] K. L. F. Bane and G. Stupakov, Terahertz radiation from a pipe with small corrugations, Nucl. Instrumen. Methods Phys. Res., Sect. A 677, 67 (2012). 